\title{
Frequency of non-alcoholic fatty liver disease in patients with type-2 diabetes mellitus and its associated risk factors
}

\author{
Shaista Kanwal ${ }^{1}$, Tahir Ghaffar ${ }^{2}$, \\ Azizul Hasan Aamir ${ }^{3}$, Khalid Usman ${ }^{4}$
}

\begin{abstract}
Objective: Non-Alcoholic Fatty Liver Disease (NAFLD) is emerging as a major public health problem globally especially in patients with Type-2 Diabetes mellitus (T2DM). This study aimed to assess the frequency of NAFLD in patients with T2DM and to study its associated risk factors.

Methods: This descriptive study was conducted from April 2020 to October 2020 at the Hayatabad Medical Complex, Peshawar. Adult patients with T2DM were included in the study and underwent abdominal ultrasound for the identification of NAFLD. All the relevant clinical and biochemical characteristics were measured.

Results: Out of 384 participants, 236 patients (61.5\%) had NAFLD on ultrasound. Patients with NAFLD had higher mean BMI, higher HbA1c, increased waist circumference, raised ALT, higher triglyceride, and low $\mathrm{HDL}$. Logistic regression analysis revealed a statistically significant association with central obesity $(\mathrm{OR}=$ $5.448,95 \% \mathrm{Cl}=1.416-20.959, p=0.014)$, higher $\mathrm{BMI}(\mathrm{OR}=4.435,95 \% \mathrm{Cl}=2.127-9.246, p<0.0001)$, higher $\mathrm{HbA} 1 \mathrm{c}[>11 \%](\mathrm{OR}=3.602,95 \% \mathrm{Cl}=1.438-9.019, p=0.006)$, and elevated ALT $(\mathrm{OR}=3.211,95 \% \mathrm{Cl}=1.509$ $6.835, p=0.002)$. The highest odds for NAFLD were found for hypertriglyceridemia $(O R=11.624,95 \% \mathrm{Cl}$ $=5.405-24.998, p<0.0001)$ and low HDL $(\mathrm{OR}=11.543,95 \% \mathrm{Cl}=2.590-51.439, p=0.001)$, respectively.

Conclusions: High frequency of NAFLD along with its associated clinical and laboratory risk factors were revealed. This underpins the significance of screening T2DM patients for NAFLD and assessment for and modification of its associated risk factors in routine clinical practice.
\end{abstract}

KEY WORDS: Type-2 Diabetes mellitus, Non-alcoholic fatty liver disease, HbA1c.

doi: https://doi.org/10.12669/pjms.37.5.4211

How to cite this:

Kanwal S, Ghaffar T, Aamir AH, Usman K. Frequency of non-alcoholic fatty liver disease in patients with type-2 diabetes mellitus and its associated risk factors. Pak J Med Sci. 2021;37(5):1335-1341. doi: https://doi.org/10.12669/pjms.37.5.4211

This is an Open Access article distributed under the terms of the Creative Commons Attribution License (http://creativecommons.org/licenses/by/3.0), which permits unrestricted use, distribution, and reproduction in any medium, provided the original work is properly cited.

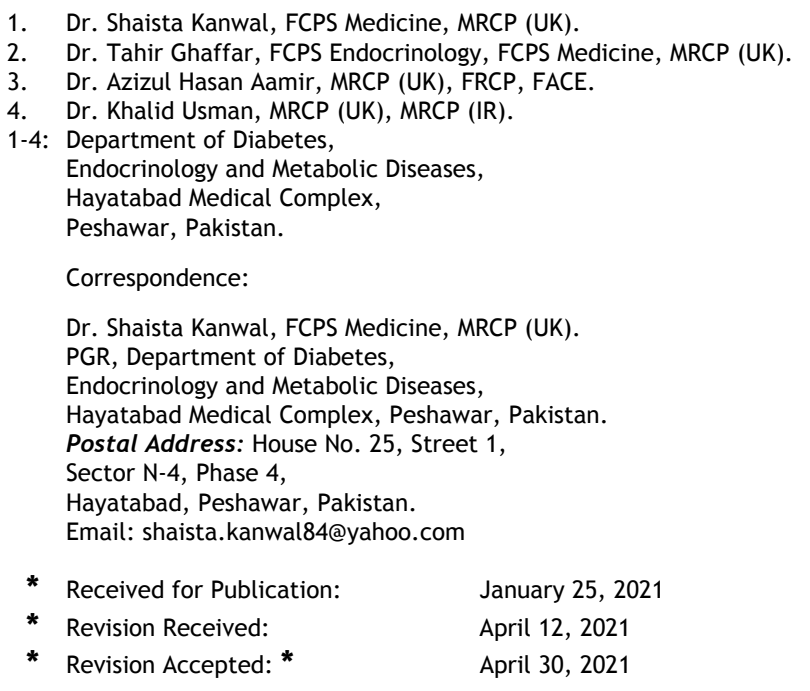

Pak J Med Sci September - October 2021

\section{INTRODUCTION}

Non-alcoholic fatty liver disease (NAFLD) comprises of a spectrum of liver diseases, ranging from fatty liver to non-alcoholic steatohepatitis (NASH) which is accompanied by inflammation. ${ }^{1}$ NAFLD is a well-known cause of Chronic liver disease (CLD), hepatocellular carcinoma and liver transplant, and is related with an increased risk of Type-2 Diabetes mellitus (T2DM), chronic kidney disease, cardiovascular disease and malignancies. ${ }^{2}$ Patients with NAFLD are found clinically to have elements of metabolic syndrome such as obesity, T2DM, hypertension and hyperlipidemia, among which T2DM is a crucial risk factor for the occurrence of NAFLD and a significant 
predictor of unfavorable clinical outcomes. ${ }^{3}$ The presence of insulin resistance and compensatory hyperinsulinemia can explain this association between NAFLD and T2DM. ${ }^{4}$

Global prevalence of NAFLD in the general public is around $15-30 \%$, however the prevalence amongst patients with obesity or T2DM is 70$80 \% .^{5}$ This supports a bidirectional relationship between T2DM and NAFLD, suggesting that these metabolic disorders share a common pathogenic mechanism. ${ }^{6}$ Moreover, the concomitant presence of T2DM and NAFLD aggravates insulin resistance, favors the promotion of dyslipidemia and makes optimal glycemic control difficult to achieve, thereby developing major adverse cardiovascular events. ${ }^{7}$ Transabdominal ultrasound scan (US) is a widely used diagnostic modality for NAFLD due its free availability, high sensitivity, low cost and non-invasive nature compared to the gold standard histological assessment which is limited in use because of invasive nature and associated complications. Although, the progressing epidemic of obesity and T2DM is accompanied by the rising incidence of NAFLD, the factors implicated for this raised prevalence of NAFLD in diabetics is only poorly studied. ${ }^{5}$ There is a rising tendency of these factors among Asian population who are genetically more susceptible to the presence of insulin resistance due to the difference in the amount and distribution of body fat from Caucasians. $^{8}$

However, in this regard very limited studies are performed in Pakistan where patients with T2DM are not screened routinely for NAFLD. In a hospital based study, the prevalence of NAFLD was $47 \%$, whereas the frequency of NAFLD and NASH among 26 diabetic patients was $75 \%$ and $22.5 \%$ respectively. ${ }^{9}$ In a study by Taseer et al. the frequency of NAFLD among patients with T2DM was $51 \% .{ }^{10}$ Another study reported $78.7 \%$ frequency of NAFLD in patients with T2DM. ${ }^{11}$ However, new data is required to appraise the different factors responsible for the occurrence of NAFLD among Type-2 diabetics. Therefore, the aim of this study was to determine the frequency of NAFLD in our Type-2 diabetic patients and to analyze the various clinical and laboratory parameters associated with the presence of NAFLD in patients with T2DM. This will not only provide valuable information about the burden of NAFLD among diabetics but will also determine its predictive risk factors, which will in turn strengthen the importance of primary prevention and prompt management.

\section{METHODS}

This descriptive cross-sectional study was conducted from April 2020 to October 2020 at the Department of Diabetes, Endocrinology and Metabolic Diseases, Hayatabad Medical Complex, Peshawar, Pakistan. The study was approved by the ethical committee of the hospital under Ref. No. 276/HEC/B\&PSC/2020 Dated 06/03/2020. The patients were selected through nonprobability consecutive sampling. Taking $51 \%$ proportion of NAFLD in patients with $\mathrm{T}_{2 \mathrm{DM}},{ }^{10} 95 \%$ confidence interval and 5\% margin of error, the calculated sample size was 384 using WHO calculator for sample size calculation. ${ }^{12}$ After getting written informed consent, adult patients with T2DM were enrolled in the study. Patients with type $1 \mathrm{DM}$, pregnancy, malignancy, history of any quantity of alcohol intake, history of intake of traditional medications, CLD, previous history of acute hepatitis of any cause including viral causes, known cases of autoimmune hepatitis, Wilson disease, hemochromatosis and alpha 1 antitrypsin deficiency were excluded from the study. Similarly, patients who had used methotrexate, corticosteroids, tamoxifen, amiodarone, low dose estrogen ( $\leq 0.3 \mathrm{mg}$ conjugated estrogen) in the last one year and those on active drug therapy for obesity were also excluded from the study.

Anthropometric measurements were obtained, and body mass index (BMI) was determined. As per WHO guidelines for the Asian people; patients with a BMI $\leq 18.5 \mathrm{~kg} / \mathrm{m}^{2}$ were labelled underweight, BMI between 18.5 to $22.9 \mathrm{~kg} / \mathrm{m}^{2}$ as of normal weight, those with a BMI between 23 to $27.4 \mathrm{~kg} / \mathrm{m}^{2}$ as overweight, whereas those with a $\mathrm{BMI} \geq 27.5 \mathrm{~kg} / \mathrm{m}^{2}$ were considered obese. ${ }^{13,14}$ Males with a waist circumference of more than $90 \mathrm{~cm}$, and females with a waist circumference of more than $80 \mathrm{~cm}$ were labelled to have central obesity. ${ }^{15}$ Diabetes Mellitus (DM) was diagnosed according to the American Diabetes Association (ADA) diagnostic criteria. ${ }^{16}$ Hypertension was defined as per the American College of Cardiology/ American Heart Association (ACC/AHA) 2017 guidelines. ${ }^{17}$ Laboratory factors like fasting plasma glucose (FBS), two hours postprandial plasma glucose (RBS), glycated hemoglobin (HbA1c), serum creatinine and uric acid, blood urea, fasting lipid profile, serum bilirubin, serum alanine aminotransferase (ALT) and alkaline phosphatase were evaluated. Serological markers of viral hepatitis (Hepatitis B and C) were also measured. Patients were considered to have dyslipidemia if 
they satisfied one of these criteria: Low density lipoprotein- cholesterol (LDL-C) $>100 \mathrm{mg} / \mathrm{dl}$, total cholesterol $>200 \mathrm{mg} / \mathrm{dl}$, triglycerides $>150 \mathrm{mg} / \mathrm{dl}$, or High-density lipoprotein-cholesterol (HDL-C) $<40 \mathrm{mg} / \mathrm{dl}$ in males and $<50 \mathrm{mg} / \mathrm{dl}$ in females. Similarly, patients with triglycerides of $>150 \mathrm{mg} /$ dl were considered to have hypertriglyceridemia. ${ }^{15}$ Using NHANES III criteria, males with ALT > $40 \mathrm{IU} / \mathrm{L}$ and females with ALT > $31 \mathrm{IU} / \mathrm{L}$ were considered to have elevated ALT. ${ }^{18}$

To identify fatty changes in the liver, all study participants underwent abdominal ultrasonography utilizingahigh-resolution B-mode ultrasound system. To prevent interpersonal variation, all ultrasounds were performed by a single experienced radiologist. Increased hepatic echogenicity in comparison to spleen and kidney, with diminution of the wave, waning of demarcation of the diaphragm, and inadequate delineation of the intra hepatic framework were used to detect NAFLD on ultrasound. One or two of these criteria were required to be accomplished to prevent false positive results. ${ }^{19}$

Statistical Analysis: Statistical analysis was accomplished by means of SPSS version 20. Quantitative data were analyzed for mean \pm standard deviation, whereas percentages were calculated for categorical variables. Patients with and without NAFLD were compared. Categorical variables and quantitative variables between the two groups were compared by performing chi-square test and independent sample's t-test, respectively. Various clinical and laboratory features associated with the occurrence of NAFLD

Table-I: Comparison of baseline clinical characteristics of Type-2 DM patients with and without NAFLD.

\begin{tabular}{|c|c|c|c|c|c|}
\hline \multicolumn{2}{|l|}{ Patient Characteristic } & $\begin{array}{l}\text { All Patients } \\
(n=384)\end{array}$ & $\begin{array}{l}\text { Without NAFLD } \\
\qquad(n=148)\end{array}$ & $\begin{array}{l}\text { With NAFLD } \\
\quad(n=236)\end{array}$ & p-value \\
\hline \multicolumn{2}{|l|}{ Age (years) } & $55.22 \pm 7.75$ & $54.41 \pm 7.40$ & $55.73 \pm 7.93$ & 0.1 \\
\hline \multirow{2}{*}{ Gender } & Male & $166(43.2 \%)$ & $70(47.3 \%)$ & $96(40.7 \%)$ & \multirow{2}{*}{0.2} \\
\hline & Female & $218(56.78 \%)$ & $78(52.7 \%)$ & $140(59.3 \%)$ & \\
\hline \multicolumn{2}{|l|}{ BMI $\left(\mathrm{Kg} / \mathrm{m}^{2}\right)$} & $27.72 \pm 4.7936$ & $24.555 \pm 3.3012$ & $29.708 \pm 4.5117$ & \multirow[t]{2}{*}{$<0.0001$} \\
\hline \multirow{4}{*}{$\begin{array}{l}\text { BMI Categories } \\
\left(\mathrm{kg} / \mathrm{m}^{2}\right)\end{array}$} & $<18.5$ & $4(1.04 \%)$ & $4(2.7 \%)$ & $0(0 \%)$ & \\
\hline & $18.5-22.9$ & $61(15.9 \%)$ & $45(30.4 \%)$ & $16(6.8 \%)$ & \multirow{3}{*}{$<0.0001$} \\
\hline & 23-27.4 & $133(34.6 \%)$ & $70(47.3 \%)$ & $63(26.7 \%)$ & \\
\hline & $\geq 27.5$ & $186(48.4 \%)$ & $29((19.6 \%)$ & $157(66.5 \%)$ & \\
\hline \multicolumn{2}{|c|}{ Waist Circumference (cm) } & $105.76 \pm 15.214$ & $94.39 \pm 11.012$ & $112.89 \pm 12.996$ & $<0.0001$ \\
\hline \multirow{2}{*}{ Central Obesity } & No & $44(11.5 \%)$ & $40(27.02 \%)$ & $4(1.69 \%)$ & \multirow{2}{*}{$<0.0001$} \\
\hline & Yes & $340(88.5 \%)$ & $108(72.97 \%)$ & $232(98.31 \%)$ & \\
\hline \multirow{2}{*}{ Smoking } & No & $284(73.96 \%)$ & $128(13.5 \%)$ & $156(33.9 \%)$ & \multirow{2}{*}{$<0.0001$} \\
\hline & Yes & $100(26.04 \%)$ & $20(86.5 \%)$ & $80(66.1 \%)$ & \\
\hline \multicolumn{2}{|c|}{ Duration of Type 2 DM (years) } & $12.87 \pm 6.347$ & $11.74 \pm 6.089$ & $13.58 \pm 6.415$ & \multirow[t]{2}{*}{0.006} \\
\hline \multirow{4}{*}{$\begin{array}{l}\text { Duration of Type-2 } \\
\text { DM categories (years) }\end{array}$} & $<5$ & $35(9.1 \%)$ & $15(10.1 \%)$ & $20(8.5 \%)$ & \\
\hline & $5-10$ & $61(15.9 \%)$ & $32(21.6 \%)$ & $29(12.3 \%)$ & \multirow{3}{*}{0.004} \\
\hline & $10-15$ & $144(37.5 \%)$ & $61(41.2 \%)$ & $83(35.2 \%)$ & \\
\hline & $>15$ & $144(37.5 \%)$ & $40(27.03 \%)$ & $104(44.07 \%)$ & \\
\hline \multirow{2}{*}{ Hypertension } & No & $137(35.7 \%)$ & $82(55.4 \%)$ & $55(23.3 \%)$ & \multirow{2}{*}{$<0.0001$} \\
\hline & Yes & $247(64.3 \%)$ & $66(44.6 \%)$ & $181(76.7 \%)$ & \\
\hline \multicolumn{2}{|l|}{ Systolic BP (mmHg) } & $136.07 \pm 17.742$ & $129.19 \pm 17.203$ & $140.38 \pm 16.716$ & $<0.001$ \\
\hline \multicolumn{2}{|l|}{ Diastolic BP (mmHg) } & $83.19 \pm 9.724$ & $79.19 \pm 8.769$ & $85.70 \pm 9.467$ & $<0.001$ \\
\hline
\end{tabular}


were evaluated by performing multivariate logistic regression analysis. Each of independent variable was analyzed for odds ratio (ORs) along with its 95\% confidence intervals (CIs). P-value of less than 0.05 was considered statistically significant.

\section{RESULTS}

A total of 384 participants were enrolled for the study, of whom there were $166(43.23 \%)$ males and $218(56.77 \%)$ females. The mean age, BMI, waist circumference and mean duration of T2DM are presented in Table-I. Overall, NAFLD was present in $236(61.5 \%)$ study participants. Moreover, patients having NAFLD were compared with patients having no ultrasonographic evidence of NAFLD. There was no statistically significant difference between the two groups regarding mean age and gender distribution. Though, there was a statistically significant difference amongst the two groups in terms of mean BMI, waist circumference, T2DM duration, systolic and diastolic BP, HbA1c, triglycerides, total cholesterol, LDL and HDL cholesterol, serum uric acid and ALT. The baseline demographic and laboratory characteristics along with the comparison of clinical and laboratory findings between the two groups of patients are presented in Table-I and Table-II, respectively.

The results of the multivariate logistic regression analysis are shown in Table-III. These results showed that smoking, hypertension, central obesity, obesity, higher HbA1c ( $\geq 11 \%$ ), elevated ALT, low HDL and hypertriglyceridemia were having independent association with the presence of NAFLD on ultrasound in T2DM patients. High triglyceride level and low HDL levels were the variables with the strongest association, conferring 11.6- and 11.5-fold increased likelihood of NAFLD in T2DM patients, respectively.

Table-II: Comparison of laboratory parameters of Type-2 DM patients with and without NAFLD.

\begin{tabular}{|c|c|c|c|c|c|}
\hline \multicolumn{2}{|l|}{ Lab Parameter } & $\begin{array}{c}\text { All patients } \\
(n=384)\end{array}$ & $\begin{array}{c}\text { Without NAFLD } \\
\quad(n=148)\end{array}$ & $\begin{array}{l}\text { With NAFLD } \\
\quad(n=236)\end{array}$ & $p$-value \\
\hline \multicolumn{2}{|l|}{ FBS (mg/dl) } & $184.17 \pm 73.311$ & $161.53 \pm 62.401$ & $198.36 \pm 76.148$ & $<0.0001$ \\
\hline \multicolumn{2}{|l|}{ RBS (mg/dl) } & $260.35 \pm 87.799$ & $232.12 \pm 75.703$ & $278.05 \pm 90.345$ & $<0.0001$ \\
\hline \multicolumn{2}{|l|}{ HbA1c (\%) } & $11.345 \pm 2.3153$ & $10.134 \pm 2.0455$ & $12.105 \pm 2.1483$ & $<0.0001$ \\
\hline \multirow{3}{*}{ HbA1c Categories (\%) } & $7-8.9$ & $75(19.5 \%)$ & $49(33.1 \%)$ & $26(11.01 \%)$ & \multirow{3}{*}{$<0.0001$} \\
\hline & $9-10.9$ & $88(22.9 \%)$ & $50(33.8 \%)$ & $38(16.1 \%)$ & \\
\hline & $\geq 11$ & $221(57.6 \%)$ & $49(33.1 \%)$ & $172(72.9 \%)$ & \\
\hline \multicolumn{2}{|c|}{ Total cholesterol (mg/dl) } & $174.11 \pm 117.565$ & $140.38 \pm 32.807$ & $195.26 \pm 143.829$ & $<0.0001$ \\
\hline \multicolumn{2}{|l|}{ Triglycerides (mg/dl) } & $206.90 \pm 100.638$ & $134.20 \pm 45.280$ & $252.50 \pm 99.031$ & $<0.0001$ \\
\hline \multicolumn{2}{|l|}{ LDL (mg/dl) } & $98.46 \pm 34.627$ & $80.01 \pm 21.465$ & $110.02 \pm 36.296$ & $<0.0001$ \\
\hline \multicolumn{2}{|l|}{ HDL (mg/dl) } & $30.70 \pm 10.479$ & $36.06 \pm 10.842$ & $27.33 \pm 8.714$ & $<0.0001$ \\
\hline \multicolumn{2}{|l|}{ Serum uric acid (mg/dl) } & $4.402 \pm 1.5109$ & $3.602 \pm 1.3424$ & $4.903 \pm 1.3921$ & $<0.0001$ \\
\hline \multicolumn{2}{|c|}{ Alanine transaminase (ALT) (U/L) } & $31.26 \pm 17.697$ & $22.52 \pm 13.613$ & $36.74 \pm 17.78$ & $<0.0001$ \\
\hline \multirow{2}{*}{ ALT categories } & Normal & $232(60.4 \%)$ & $121(81.8 \%)$ & $111(47.0 \%)$ & \multirow{2}{*}{$<0.0001$} \\
\hline & Elevated & $152(39.6 \%)$ & $27(18.2 \%)$ & $125(53.0 \%)$ & \\
\hline \multirow{2}{*}{ Dyslipidemia } & No & $63(16.4 \%)$ & $57(38.5 \%)$ & $6(2.5 \%)$ & \multirow{2}{*}{$<0.0001$} \\
\hline & Yes & $321(83.6 \%)$ & $91(61.5 \%)$ & $230(97.5 \%)$ & \\
\hline \multirow{2}{*}{ Low HDL } & No & $22(5.7 \%)$ & $18(12.2 \%)$ & $4(1.7 \%)$ & \multirow{2}{*}{$<0.0001$} \\
\hline & Yes & $362(94.3 \%)$ & $130(87.8 \%)$ & $232(98.3 \%)$ & \\
\hline \multirow{2}{*}{ Hypertriglyceridemia } & No & $134(34.9 \%)$ & $110(74.3 \%)$ & $24(10.2 \%)$ & \multirow{2}{*}{$<0.0001$} \\
\hline & Yes & $250(65.1 \%)$ & $38(25.7 \%)$ & 212 (89.8\%) & \\
\hline
\end{tabular}

Pak J Med Sci $\quad$ September - October $2021 \quad$ Vol. $37 \quad$ No. $5 \quad$ www.pjms.org.pk 1338 
NAFLD in T2 Diabetes Mellitus Patients

Table-III: Logistic Regression analysis for risk factors associated with NAFLD in patients with Type-2 DM.

\begin{tabular}{|c|c|c|c|c|}
\hline Variable & & Odds Ratio (OR) & $95 \%$ confidence interval & $p$-value \\
\hline \multirow{2}{*}{ Gender } & Male & 1.0 & & \\
\hline & Female & 1.582 & $0.670-3.736$ & 0.296 \\
\hline \multirow{2}{*}{ Smoking } & No & 1.0 & & \\
\hline & Yes & 5.232 & $1.784-15.340$ & 0.003 \\
\hline \multirow{2}{*}{ Hypertension } & No & 1.0 & & \\
\hline & Yes & 2.221 & $1.111-4.438$ & 0.024 \\
\hline \multirow{2}{*}{ Central obesity } & No & 1.0 & & \\
\hline & Yes & 5.448 & $1.416-20.959$ & 0.014 \\
\hline \multirow[t]{3}{*}{ BMI Categories $\left(\mathrm{kg} / \mathrm{m}^{2}\right)$} & $<27.5$ (Non-Obese) & 1.0 & & \\
\hline & $\geq 27.5$ (Obese) & 4.435 & $2.127-9.246$ & $<0.0001$ \\
\hline & $7-8.9$ & 1.0 & & \\
\hline \multirow[t]{2}{*}{ HbA1c categories (\%) } & $9-10.9$ & 1.616 & $0.587-4.455$ & 0.35 \\
\hline & $\geq 11$ & 3.602 & $1.438-9.019$ & 0.006 \\
\hline \multirow{2}{*}{ ALT } & Normal & 1.0 & & \\
\hline & Elevated & 3.211 & $1.509-6.835$ & 0.002 \\
\hline \multirow{2}{*}{ HDL } & Normal & 1.0 & & \\
\hline & Reduced & 11.543 & $2.590-51.439$ & 0.001 \\
\hline \multirow{2}{*}{ Triglyceride } & Normal & 1.0 & & \\
\hline & Elevated & 11.624 & $5.405-24.998$ & $<0.0001$ \\
\hline \multirow{2}{*}{ Dyslipidemia } & No & 1.0 & & \\
\hline & Yes & 1.773 & $0.580-5.426$ & 0.315 \\
\hline
\end{tabular}

\section{DISCUSSION}

Around $61.5 \%$ of the study participants with T2DM satisfied the ultrasound criteria for NAFLD. This suggests a higher burden of NAFLD in patients with T2DM in our local population. The frequency of NAFLD in the South East Asian population ranges from $9 \%$ to $45 \%$, and in patients with T2DM it varies from $6 \%$ to $62 \% .^{20}$ The $61.5 \%$ frequency of NAFLD in T2DM patients in the present study is very similar to that reported by a study conducted by Herath et al. in subjects having T2DM in Sri Lanka (61.9\%). ${ }^{5}$

Patients who had NAFLD had higher values of mean systolic and diastolic blood pressure, mean FBS, mean RBS, LDL cholesterol, triglycerides and HbA1c compared to those without NAFLD. These were consistent with the findings demonstrated by Dai et al. and Butt et al. in their studies., ${ }^{4,8}$ Majority of the patients who had NAFLD were smokers and on logistic regression analysis, it was evident that smoking conferred an approximately five- fold higher risk of NAFLD. These findings were comparable to those by Agarwal et al. who demonstrated that patients in the NAFLD group had a higher frequency of hypertension and smoking. ${ }^{21}$

The mean BMI of patients in this study in the NAFLD group was $29.7( \pm 4.5) \mathrm{kg} / \mathrm{m}^{2}$ compared to $24.5( \pm 3.3) \mathrm{kg} / \mathrm{m}^{2}$ in the non-NAFLD group. A study conducted by Targher et al. in T2DM patients revealed that patients with NAFLD had a mean BMI of $28.3 \mathrm{~kg} / \mathrm{m}^{2}$ compared to $26.5 \mathrm{~kg} / \mathrm{m}^{2}$ in patients with no NAFLD. These results were closely related to the mean BMI in this study. ${ }^{22}$ This study also reported a very high mean waist circumference $(112.89 \pm 12.996)$ in the study participants having NAFLD compared to those with no evidence of NAFLD (94.39 \pm 11.012$)$. Similar associations with central obesity have been described in some earlier studies as well. ${ }^{4,5}$ Moreover, obesity $(\mathrm{OR}=4.435$, $95 \% \mathrm{CI}=2.127-9.246)$ and central obesity $(\mathrm{OR}=$ $5.448,95 \% \mathrm{CI}=1.416-20.959)$ were independently associated with NAFLD. This was also observed in a study by Leite et al. who revealed that obesity 
and central obesity were independent predictive factors for NAFLD in the diabetic population. ${ }^{23}$

Studies have shown that elevated ALT could act as an independent component in the occurrence of metabolic complications. ${ }^{24}$ Overall, in the current study, 152 (39.6\%) participants had elevated ALT and those having NAFLD had a higher proportion of participants with raised ALT compared to those without NAFLD $(53.0 \%$ versus $18.2 \%, p<0.0001)$. This is in contrast to the $16.4 \%$ frequency of elevated ALT in Type-2 diabetic patients with NAFLD in a study conducted by Butt et al. ${ }^{8}$ This difference in the proportion of raised ALT between this study and our study could be due to that patients included in our study had a relatively longer duration of T2DM, had greater mean BMI, higher mean waist circumference and higher mean HbA1c. Moreover, Butt et al. conducted their study on patients with newly diagnosed T2DM in comparison to this study where majority $(44.07 \%)$ of subjects with NAFLD had T2DM for longer than 15 years. In addition, mean HbA1c in the NAFLD group in our study was $12.105 \pm 2.1483$ compared to their study where mean $\mathrm{HbA1c}$ was $8.13 \pm 1.69 .{ }^{8}$ Logistic regression analysis also demonstrated that raised $\operatorname{ALT}(\mathrm{OR}=3.211,95 \% \mathrm{CI}=1.509-6.835)$ and $\mathrm{HbA} 1 \mathrm{c}$ of $\geq 11 \%(\mathrm{OR}=3.602,95 \% \mathrm{CI}=1.438-9.019) \mathrm{had}$ independent associations with the development of NAFLD amongst T2DM patients. This finding is comparable to another study where ALT was associated significantly with the possibility of NAFLD. ${ }^{25}$ Likewise, a study by Ma et al. revealed the association of HbA1c with NAFLD. ${ }^{26}$

Another remarkable finding of our study was that $89.8 \%$ of the patients with NAFLD had hypertriglyceridemia, $88.1 \%$ of patients had both hypertriglyceridemia and central obesity and $98.3 \%$ of the patients were having reduced HDL. These results are comparable to the findings presented by Butt et al. in their study who reported that odds of NAFLD with both central obesity and high triglycerides was 3.7. ${ }^{8}$ A study by Radu et al. demonstrated that central obesity and hypertriglyceridemia had higher odds ratio for NAFLD, concluding that these factors were independent determinants of NAFLD. ${ }^{27}$

These findings are quite alarming as it is known that increased waist circumference along with high plasma triglyceride level are important cardiovascular risk factors and serves as strong determinants of cardiovascular disease. ${ }^{28}$ Our study revealed that central obesity, higher BMI, higher HbA1c, hypertriglyceridemia, low HDL levels, smoking and raised ALT were the elements having significant association with the presence of NAFLD. These results open new avenues for treatment in T2DM patients by focusing not only on glycemic control but also targeting these various cardiometabolic risk factors.

Limitations of the study: Although this study exclusively studied Type-2 diabetic patients and various parameters are compared in detail between patients with and without NAFLD, it has few limitations. Firstly, confirmation with liver biopsy was not undertaken because that is invasive and was neither feasible nor cost effective in these asymptomatic patients. Secondly, majority of T2DM patients were on statins as part of multipronged approach to achieve metabolic control and it was unethical to stop their statins or exclude them just because they were on statins. Thirdly, this study was performed at a single center in a tertiary care hospital and generalization of these findings should be done once confirmed by large scale multicenter studies. Despite this, our study revealed that approximately two out of three patients with T2DM had NAFLD.

\section{CONCLUSION}

This study reported an increased frequency of NAFLD in our diabetic population and evaluated in depth the risk factors associated with NAFLD, underpinning the significance of carrying further large-scale studies to assess the effects of lifestyle modification in the form of physical activity and dietary modifications on the status of NAFLD and glycemic control. Taking in to account the results of this study, patients and their treating physicians should emphasize on the modification of the associated factors and it is also advisable to screen diabetic patients for this condition in routine clinical practice. Early detection and timely management will help promote healthy lifestyle and prevent long term complications of the condition.

Conflict of Interest: None.

Grant Support \& Financial Disclosures: None.

\section{REFERENCES}

1. Bedossa P. Current histological classification of NAFLD: Strength and limitations. Hepatol Int. 2013;7(Suppl 2):765770. doi: 10.1007/s12072-013-9446-z

2. Estes C, Razavi H, Loomba R, Younossi Z, Sanyal AJ. Modeling the epidemic of nonalcoholic fatty liver disease demonstrates an exponential increase in burden of disease. Hepatology. 2018;67(1):123-133. doi: 10.1002/hep.29466 
3. Younossi ZM, Golabi P, de Avila L, Paik JM, Srishord M, Fukui N, et al. The global epidemiology of NAFLD and NASH in patients with type 2 diabetes: A systematic review and meta-analysis. J Hepatol. 2019;71(4):793-801. doi: 10.1016/j.jhep.2019.06.021

4. Dai W, Ye L, Liu A, Wen SW, Deng J, Wu X, et al. Prevalence of nonalcoholic fatty liver disease in patients with type 2 diabetes mellitus: A meta-analysis. Medicine (Baltimore). 2017;96(39):e8179. doi: 10.1097/MD.0000000000008179

5. Herath HMM, Kodikara I, Weerarathna TP, Liyanage G. Prevalence and associations of non-alcoholic fatty liver disease (NAFLD) in Sri Lankan patients with type 2 diabetes: A single center study. Diabetes Metab Syndr. 2019;13(1):246250. doi: $10.1016 /$ j.dsx.2018.09.002

6. Lonardo A, Ballestri S, Guaraldi G, Nascimbeni F, Romagnoli D, Zona S, et al. Fatty liver is associated with an increased risk of diabetes and cardiovascular disease - Evidence from three different disease models: NAFLD, HCV and HIV. World J Gastroenterol. 2016;22(44):96749693. doi: $10.3748 /$ wjg.v22.i44.9674

7. Targher G. Is it time for non-alcoholic fatty liver disease screening in patients with type 2 diabetes mellitus? Hepatobiliary Surg Nutr. 2020;9(2):239-241. doi: 10.21037/ hbsn.2019.10.21

8. Butt AS, Hamid S, Haider Z, Sharif F, Salih M, Awan S, et al. Nonalcoholic fatty Liver Diseases Among Recently Diagnosed Patients with Diabetes Mellitus and Risk Factors. Euroasian J Hepatogastroenterol. 2019;9(1):9-13. doi: 10.5005/jp-journals-10018-1288

9. Shah AS, KhanS, Rahim H, Chishti KA, Khan AG. Prevalence of nonalcoholic fatty liver and non-alcoholic steatohepatitis in Peshawar Cantonment, Khyber Pakhtunkhwa, Pakistan. Pak J Pharm Sci. 2018;31(1):193-198.

10. Taseer IH, Hussain L, Safdar S, Mirbahar AM, Ahmad I. Frequency of non alcoholic fatty liver disease (NAFLD) and its biochemical derangements in Type-2 diabetic patients. Pak J Med Sci. 2009;25(5):817-820.

11. Rashid A, Zafar S, Bashir A, Bakht K, Bhalli AU. Presence of non-alcoholic fatty liver disease in patients of uncontrolled and controlled type 2 diabetes mellitus. Pak Armed Forces Med J. 2019;69(4):822-825.

12. STEPS Sample Size Calculator and Sampling Spreadsheet [Internet]. WHO; 2021. Available from: https://www.who. int/ncds/surveillance/steps/resources/sampling/en/

13. WHO Expert Consultation. Appropriate body-mass index for Asian populations and its implications for policy and intervention strategies. Lancet. 2004;363(9403):157-163. doi: 10.1016/S0140-6736(03)15268-3

14. National Institute for Health and Care Excellence. BMI: preventing ill health and premature death in black, Asian and other minority ethnic groups. UK; 2013.

15. International Diabetes Federation. The IDF consensus worldwide definition of the metabolic syndrome. Brussels, Belgium: International Diabetes Federation; 2006.

16. American Diabetes Association. 2. Classification and Diagnosis of Diabetes: Standards of Medical Care in Diabetes-2020. Diabetes Care. 2020;43(Suppl 1):S14-S31. doi: $10.2337 /$ dc20-S002

17. Whelton PK, Carey RM, Aronow WS, Casey DE Jr, Collins KJ, Dennison Himmelfarb C, et al. 2017 ACC/AHA/ AAPA/ABC/ACPM/AGS/APhA/ASH/ASPC/NMA/ PCNA Guideline for the prevention, detection, evaluation, and management of high blood pressure in adults: A Report of the American College of Cardiology/American Heart Association Task Force on Clinical Practice Guidelines. Hypertension. 2018;71(6):e13-e115. doi: 10.1161/ HYP.0000000000000065
18. Gunter EW, Lewis BG, Koncikowski SM. Laboratory Procedures Used for the Third National Health and Nutrition Examination Survey (NHANES III), 1988-1994. US Department of Health and Human Services; 1996.

19. Ezzat WM, Ragab S, Ismail NA, Elhosary YA, Abd ElBaky AMNE, Farouk H, et al. Frequency of non-alcoholic fatty liver disease in overweight/obese children and adults: Clinical, sonographic picture and biochemical assessment. J Genet Eng Biotechnol. 2012;10(2):221-227. doi: 10.1016/j. jgeb.2012.05.006

20. Farrell GC, Wong VW, Chitturi S. NAFLD in Asia - as common and important as in the West. Nat Rev Gastroenterol Hepatol. 2013;10(5):307-318. doi: 10.1038/nrgastro.2013.34

21. Agarwal AK, Jain V, Singla S, Baruah BP, Arya V, Yadav R, et al. Prevalence of non-alcoholic fatty liver disease and its correlation with coronary risk factors in patients with type 2 diabetes. J Assoc Physicians India. 2011;59:351-354.

22. Targher G, Bertolini L, Padovani R, Rodella S, Tessari R, Zenari $\mathrm{L}$, et al. Prevalence of nonalcoholic fatty liver disease and its association with cardiovascular disease among type 2 diabetic patients. Diabetes Care. 2007;30(5):1212-1218. doi: $10.2337 /$ dc06-2247

23. Leite NC, Salles GF, Araujo AL, Villela-Nogueira CA, Cardoso CR. Prevalence and associated factors of nonalcoholic fatty liver disease in patients with type- 2 diabetes mellitus. Liver Int. 2009;29(1):113-119. doi: 10.1111/j.14783231.2008.01718.x

24. Krishnan A, Venkataraman J. Prevalence of nonalcoholic fatty liver disease and its biochemical predictors in patients with type-2 diabetic mellitus. Exp Clin Hepatol. 2011;7(3-4):7-10.

25. Trojak A, Walus-Miarka M, Wozniakiewicz E, Malecki MT, Idzior-Walus B. Nonalcoholic fatty liver disease is associated with low HDL cholesterol and coronary angioplasty in patients with type 2 diabetes. Med Sci Monit. 2013;19:11671172. doi: 10.12659/MSM.889649

26. Ma H, Xu C, Xu L, Yu C, Miao M, Li Y. Independent association of $\mathrm{HbA1c}$ and nonalcoholic fatty liver disease in an elderly Chinese population. BMC Gastroenterol. 2013;13:3. doi: 10.1186/1471-230X-13-3

27. Radu C, Grigorescu M, Crisan D, Lupsor M, Constantin D, Dina L. Prevalence and associated risk factors of nonalcoholic fatty liver disease in hospitalized patients. J Gastrointestin Liver Dis. 2008;17(3):255-260.

28. Sam S, Haffner S, Davidson MH, D’ Agostino RB, Feinstein $S$, Kondos G, et al. Hypertriglyceridemic waist phenotype predicts increased visceral fat in subjects with type 2 diabetes. Diabetes Care. 2009;32(10):1916-1920. doi: 10.2337/ dc09-0412

\section{Author's Contribution:}

SK conceived, designed, did literature review, performed statistical analysis \& drafted the manuscript.

TG participated in analysis and interpretation of data, and helped in drafting the manuscript.

AHA conceived, designed, and critically revised the manuscript.

KU helped in analysis and interpretation of data, and critically revised the manuscript.

All authors provided final approval for publication of the manuscript, and are responsible for the integrity of the study. 